\title{
Vernier offset produced by rotary target motion
}

\author{
LEONARD MATIN and KENNETH R. BOFF \\ Columbia University, New York, 10027 \\ and \\ JORDAN POLA \\ SUNY College of Optometry New York, New York 10010
}

\begin{abstract}
Two separated colinear lines appear displaced from colinearity when either the target or the subject's head is rotated in a frontal plare. The direction of perceived offset is reversed for opposite directions of rotation. The present experiments prove that the effect depends on some property of the visual system that is responsive to stimulus motion per se and is not manifested in the response to stationary targets. Two mechanisms which may be responsible for the rotation-contingent effect are considered: (1) An induction mechanism based on the dynamics of induced tilt or of figural aftereffect displacement. (2) A mechanism based on variation of visual latency with stimulus energy/time.
\end{abstract}

When an observer slowly rotates his head from shoulder to shoulder in a frontal plane while viewing a pair of colinear lines, the lines appear to be displaced from each other along an axis perpendicular to their lengths. When the head rotates toward the left shoulder, the top line appears displaced to the right; similarly, when the head rotates toward the right shoulder, the top line appears displaced to the left. Thus, the apparent vernier offset is in the direction opposite to the head rotation. This phenomenon is apparently universal, with more than 50 observers having reported it. Since a similar offset is observed when the pair of lines is rotated in the frontal plane of observers with stationary heads (Figure 1), the role that vestibular influences play in the illusion with rotating head is likely to be minimal at best, and the primary determinants must be intrinsic to the retinal stimulus.

The simplest interpretation for this effect is the following: The offset required for an appearance of colinearity with stationary targets undergoes a systematic change with the orientation of the target on the reti.1a. A rotating target should then appear to undergo a systematic change in offset that corresponds to the sequence of offsets perceived with change in the orientation of the stationary target. This implies that what is significant in the production of the effect is the series of retinal orientations stimulated by the target rather than the fact of the

This research was supported by National Science Foundation Research Center BMS73-01463 and PHS Research Grant EY 00375 from the National Eye Institute, National Institutes of Health. We would like to thank Drs. Ilene Bernstein and Richard McConchie, who assisted in the collection of data in the unlimited viewing condition. A report of these experiments was presented at the annual meeting of the Eastern Psychological Association, New York City, April 1975 (Boff, Matin, \& Pola, Note 1). retinal motion per se and will be referred to as the "sequential positions hypothesis."

The experiments reported here were conducted to investigate this hypothesis. We have measured the perceived offset for vernier targets at different orientations within a frontal plane under a variety of conditions, and compared these with measurements of the perceived offset obtained with the rotating target.

\section{METHOD}

Two sets of experiments investigating the perceived of fset of stationary vernier targets as a function of frontal plane orientation were conducted several years apart. In addition, the perceived offset with rotating vernier targets was measured in conjunction with the more recent set of experiments. Both sets of experiments were conducted with completely different sets of apparatus and experimental subjects.

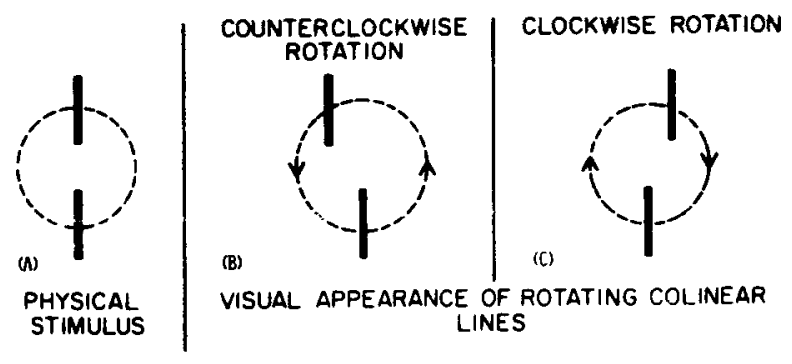

Figure 1. A pair of colinear lines (A) appears offset as in (B) when it is rotated counterclockwise about the point centered between them and as in $(C)$ when rotation is clockwise. The physical stimulus and visual appearance are shown when the target reaches a vertical position during rotation. The dashed line indicates the path of rotation; the arrows indicate direction; the magnitudes of the effects shown here in $(B)$ and $(C)$ are exaggerated for presentation to the reader. (Rotation of the head while viewing the stationary target results in an of fset whose direction is the same for a given direction of rotation of the retinal stimulus.) 


\section{Vernier Offset with Stationary Targets \\ Limited Duration Viewing}

Apparatus. The vernier targets were produced on the face of a cathode ray oscilloscope (Tektronix No. 651 with a P24 phosphor having a decay time of $1.5 \mu \mathrm{sec}$ ) driven by a Linc- 8 computer (Digital Equipment Corp.) and monocularly viewed by the subject through his natural pupil at a distance of $17.2 \mathrm{ft}$. The vernier target (see Figure 2) was composed of a pair of vertical lines (at a luminance of $4.28 \mathrm{~mL}$ ), each $24.8^{\prime}$ (visual angle) in length and $0.13^{\prime}$ in width, vertically separated by $16.6^{\prime}$. The horizontal offset between the two lines varied between trials; this was accomplished by variation of the horizontal position of the upper line relative to the fixed position of the lower line. The subject's fixation was at the center of an array of eight continuously illuminated $(3.5 \mathrm{~mL})$ points arranged in a $1.9^{\circ}$ circle that was concentric with the vernier target. A beamsplitter placed in front of the subject's viewing eye was used to combine the fixation array (reflected) and vernier target (transmitted).

Orientation of the vernier target within a frontal plane was controlled by means of a rotatable Pechan prism whose main axis was coincident with the axis of rotation and was perpendicular to the face of the oscilloscope. [The Pechan prism functions, as does a dove prism, to provide $360^{\circ}$ of image rotation for $180^{\circ}$ of prism rotation. However, since the two exterior surfaces are perpendicular to the viewing axis, optical aberrations (particularly chromatic aberrations) are minimized.] Since the center of the vernier target was coincident with the main axis of the Pechan prism, rotation of the prism produced a rotation of the target image about its own center at the observer's retina.

The vernier target was generated by means of a point-by-point digital plotting procedure governed by a subroutine of the computer program that controlled the entire experiment, including stimulus production, randomization, data collection, and tabulation. Each of the two lines of the vernier target was composed of 192 equally spaced points within $1 \frac{1}{2}$ in. on the face of the oscilloscope. A single production of the two lines took $11 \mathrm{msec}$, with the exposure duration of each point lasting for $8 \mu \mathrm{sec}$ (including rise and decay times to $10 \%$ of maximum), while the initiation of exposures for successive points was separated by $30 \mu \mathrm{sec}$. The resulting stimulus display appeared to the subject as a pair of continuous, illuminated lines which flashed simultaneously. Thus, a stimulus consisting of a single production of

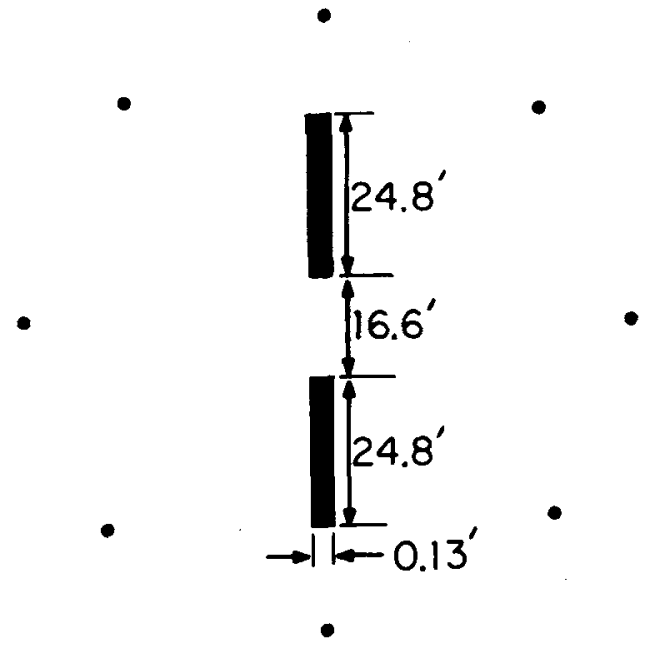

Figure 2. The vernier target employed in both the limited viewing and rotating target conditions. The physical dimensions for the vernier lines are indicated. The diameter of the fixation circle subtended $1.9^{\circ}$ arc. (Dimensions for unlimited viewing conditions are given in the text; the fixation circle was not employed in the unlimited condition.) the vernier target is referred to below as an $11-\mathrm{msec}$ stimulus. In the present experiment, three different stimulus durations were employed: $11 \mathrm{msec}, 275 \mathrm{msec}$ ( 25 repetitions of the 11 -msec line pair), and 1,003 msec ( 91 repetitions).

Procedure. Visual stimuli were presented in a totally darkened room in a trial-by-trial method of constants format. The fixating subject viewed the target through the Pechan prism with head position stabilized by means of a chinrest. The stimulus on each trial consisted of the pair of vernier lines indicated in Figure 2, with the top line being displaced horizontally in one of five positions relative to the fixed position of the bottom line. These were alternated according to a randomized block design. The subject reported on the position of the top line (left or right) relative to the bottom line by means of manual switch closures that were signaled to the computer. In each experimental session, stationary targets were presented at a single duration in each of 13 orientations equally spaced by $15^{\circ}$ steps between $0^{\circ}$ and $180^{\circ}\left(0=\right.$ horizontal; $180^{\circ}$ was a repeat of the $0^{\circ}$ condition with the variable line to the left). Within a session, the sequence of orientations was ordered either from $0^{\circ}$ to $180^{\circ}$ or from $180^{\circ}$ to $0^{\circ}$, with the two orders alternating between sessions for each subject. Two sessions were run with 50 trials per orientation per session; 10 trained observers with normal or optically corrected acuity participated in the experiment, with 8 serving in each of the three duration conditions and 2 more only in the 11 - and 275 -msec conditions. Six of the subjects viewed with their right eyes, and 4 used their left eyes.

\section{Unlimited Duration Viewing}

These experiments were conducted at an earlier date with different apparatus and procedure than was employed in the limited duration condition. The vernier targets were produced by placing masks over a diffusing glass that served as the front surface of a light box. The dimensions of the two vernier lines were different from those in Figure 2, with line length $12.5^{\prime}$, line width $40^{\prime \prime}$, vertical separation between targets $16.7^{\prime}$, and luminance $3.9 \mathrm{~mL}$. The target was positioned $18.0 \mathrm{ft}$ in front of the subject's cubicle. A rotatable dove prism through which the subject viewed the target-either with or without a $2.2-\mathrm{mm}$ artificial pupil-was mounted on the wall of the cubicle. The horizontal position of the lower line of the vernier target was set by the experimenter by means of a micrometer on each trial.

Each of the three subjects was presented with the vernier target in each of 18 orientations determined by the setting of the dove prism. On each trial, one of five horizontal offsets was presented for each target orientation. The subject was instructed to maintain fixation at the center of the vernier target (monocular viewing with the left eye only). He viewed the target for as long as he needed to make a judgment, typically taking no more than $2-3 \mathrm{sec}$. On each trial he reported whether the top line appeared to the left or to the right of the lower line. Different orientations were randomly ordered within a session, with a total of 100 trials presented at each orientation (in a randomized block design) when viewing was with or without the artificial pupil.

\section{Vernier Offset with Rotating Targets}

The data with rotating stimuli were collected during the same sessions as were the data for limited duration viewing. Hence, subjects, apparatus, and procedure are identical, except that on each trial the vernier target rotated through four complete revolutions in a 5.6-sec period of presentation.

Measurements were made separately for clockwise (cw) and for counterclockwise (ccw) rotation. Both directions were employed in each session, with a block of trials in one direction of rotation followed by a block of trials in the other direction. Subjects were instructed to report on the position of the top line relative to the lower line as the stimulus passed through the vertical. Data for 200 trials ( 40 trials per offset presented according to a randomized blocks design) were collected for each direction of rotation. 


\section{RESULTS}

No systematic differences were observed in the individual data between subjects using their left or their right eyes in either the stationary (limited viewing) or rotating target conditions. Hence, data for left and right eyes are not treated separately.

\section{Stationary Targets-Limited Viewing Conditions}

Median points of subjective equality (PSEs) for colinearity across subjects are plotted in Figure 3 as a function of target orientation. [All PSEs were calculated on the Linc- 8 computer from ogives fitted to the psychometric functions by means of the probit method (Finney, 1947); they are the $50 \%$ points on the ogives.] Although there is some tendency for more oblique orientations to yield larger median PSEs than those near the horizontal and vertical, the trend is not nearly as systematic as the trend of the JNDs which are significantly larger at the oblique orientations (the oblique effect; see Leibowitz, 1955, for a previous report of this).

\section{Rotating Targets}

The median PSEs for the rotating targets are shown by the two large filled circles at the abscissa value corresponding to the vertical in Figure 3. As in our previous observations, clockwise rotation of the target resulted in a PSE for each subject in which the upper line must be displaced to the left for an appearance of colinearity. Similarly, counterclockwise rotation of the target resulted in a PSE for each subject in which the upper line was displaced to the right for an appearance of colinearity. The median PSEs for both directions of rotation are substantially greater than the offsets obtained with the stationary targets at any orientation for any duration. While the median PSEs for stationary targets for almost all orientations are such that the upper line must be displaced to the right of the lower line in order for the stimulus configuration to appear colinear to the observer, the direction of the effect for the rotating target is always symmetrical, with the upper line to the right for counterclockwise rotation and to the left for clockwise rotation. The shape of the function for the stationary targets is quite variable between subjects, although there is complete uniformity in the direction of the PSEs for the rotating targets. It is worth noting that the correlation between the PSEs for the clockwise and counterclockwise conditions is -0.78 .

Each of the panels in Figure 4 provides a scatter diagram relating the data for the rotating target and data for one of the durations of exposure of the stationary stimulus. The range of PSEs obtained for the stationary target across all orientations are plotted for each subject on the abscissa while the range obtained for the two directions of rotation (equal to the difference between the two PSEs) is plotted on the ordinant. The strong component of

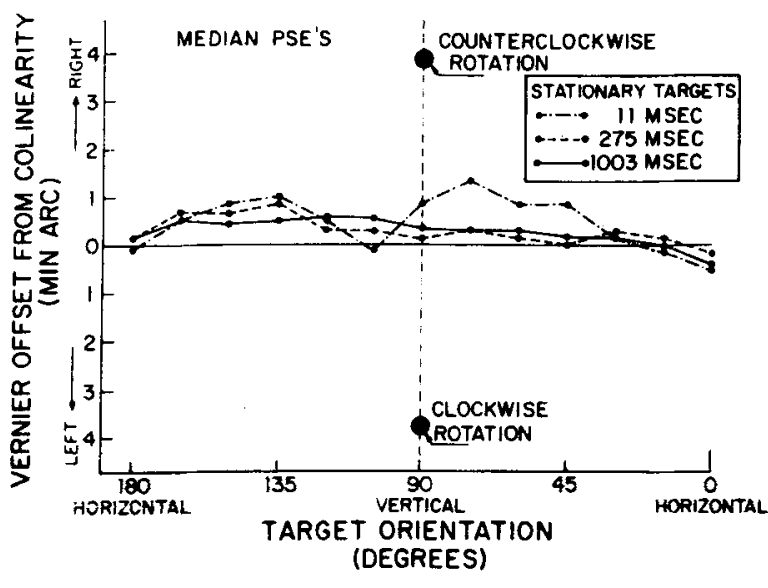

Figure 3. Median PSEs for conditions of limited duration viewing of stationary targets are plotted as a function of target orientation from $0^{\circ}$ through $180^{\circ}$ in $15^{\circ}$ steps for each of three stimulus durations: 11, 275, and 1,003 msec. Each PSE represents a position of offset (left or right) between the vernier lines, which appeared to the subjects as being colinear. The solid dark circles at the vertical meridian represent the median PSEs of the same subjects for the counterclockwise and clockwise rotating targets. respectively.

linearity in the relation between the two ranges in each panel is visible in the diagram and in the substantial positive values of the Pearson productmoment correlation, which indicate that $60 \%$ to $86 \%$ of the variance of the two measures among individuals is common to both. As one might expect from Figure 3, however, the slope of the linear relation far exceeds 1 (equality of the two ranges), with the range of PSEs for the rotating target exceeding the range for the stationary target for all subjects under each condition (with one exception).

\section{Stationary Targets-Unlimited Viewing Conditions}

Figure 5 shows the PSEs for each of three subjects in the unlimited viewing condition as a function of target orientation. Although the functions for the three subjects differ markedly from each other (as do the functions for the different subjects in the limited duration condition), and although substantial differences do appear at some points, the data for each subject are not substantially different when viewing was through the $2.2-\mathrm{mm}$ artificial pupil or the natural pupil.

\section{DISCUSSION}

\section{The Sequential Positions Hypothesis Fails}

The present report describes several experiments designed to deal with the sequential positions hypothesis as an interpretation of the rotation-contingent illusion of vernier offset. This hypothesis provides the simplest interpretation to the illusion. The sequential positions hypothesis suggests that the observations made on the rotating target are a consequence of differences in perceived offset at differ- 


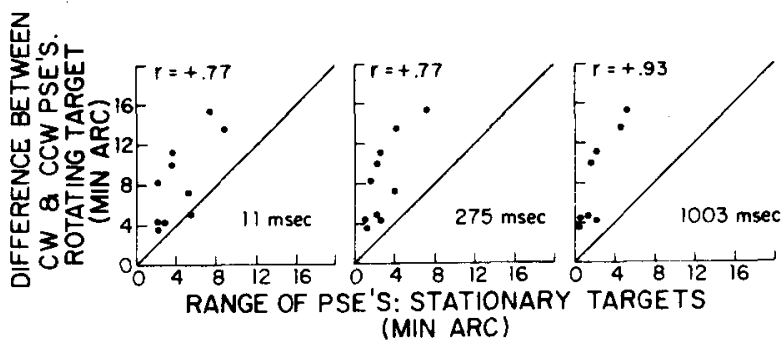

Figure 4. The range of PSEs for the rotating target as a function of the range of PSEs for the stationary targets for each of three durations of presentation of the stationary target: 11,275 , and 1,003 msec. Each point represents the data from one subject. The range of PSEs for the rotating targets is calculated as the difference between PSEs for counterclockwise and clockwise rotation. (For counterclockwise rotation, the upper line of the target must be offset to the right of the lower line for the lines to appear colinear to the subject. Similarly, for clockwise rotation, the offset of the upper line of the target must be to the left.) The range of PSEs for the stationary targets is the difference between the maximum offset to the right and the maximum offset to the left. The Pearson product-moment correlation, $r$, is indicated in each panel.

ent frontal plane orientations as we have measured them with stationary vernier targets. Support for this hypothesis is provided by some aspects of the correlations between the rotation-contingent illusion and the PSEs measured with stationary targets (Figure 4). Nevertheless, there are several substantial reasons for concluding that the rotation-contingent effect and the results with stationary targets are not merely different manifestations of the same process: (1) the magnitude of the vernier offset for the rotating target is much greater than the offset at any orientation measured with the stationary target (Figure 3) or of the difference in offsets between any pair of orientations; (2) the direction of the illusion is reversed for opposite directions of rotation for all subjects although the sequential positions hypothesis predicts that the effect be identical for both directions of rotation; (3) the direction of the rotation-contingent offset is identical for all subjects for a given direction of rotation, whereas the stationary target PSEs for different subjects undergo very different functions of target orientation (cf. Figure 5). Both of these observations conflict with the simple view that the illusion with the moving target results from a systematic change in appearance at different orientations that conforms to the change in PSE measured with the stationary target. Further, the possibility that the subject either "averages" or "picks the largest" stationary offset or difference in of fsets as the offset perceived during rotation (a modification of the sequential positions hypothesis) is ruled out by both observations.

Any simple development of the sequential positions hypothesis thus appears to fail. Nor can we consider dioptric factors in the eye as of any substantial and basic importance. For, although we may describe both the movement-contingent illusion and the offsets with stationary targets by saying that it is "as if" a "differential wedge prism" were introduced between the upper and lower lines, no substantial difference was obtained between the stationary conditions with and without an artificial pupil. Since only paraxial rays of light are transmitted through an artificial pupil, the effects of accommodative changes on the quality of the image are reduced, and hence any influences due to optical aberrations are minimized. If a "differential prism" did reside in the dioptrics of the eye, it is likely that its actions would have been modified by the artificial pupil. Therefore, the basis of the present effect must be neural in origin.

Since the rotation-contingent illusion is not a simple consequence of the change of the stationary PSE with orientation, the illusion must involve some property of the perceptual system that responds during stimulation by movement but not during viewing of a stationary target. However, the range of the stationary and moving offsets are highly correlated among individuals, which suggests that some aspect of the mechanism involved in the production of offset for stationary targets is also involved in the production of of fset for rotating targets. ${ }^{1}$

\section{Two Possible Mechanisms Subserving Production of Offset by Motion}

We have considered two mechanisms which may be responsible for the rotation-contingent effect.

\section{Spatial Induction}

This assumes that spatial induction effects observed with stationary stimuli-either the induction of displacement [figural aftereffects (Kohler \& Wallach, 1944; see Graham, 1965, for review)] or the induction of tilt (Bouma \& Andriessen, 1970; Matin, 1974; Matin, Matin, Hirsch, \& Kornheiser, 1972)-provide the basis for the rotation-contingent

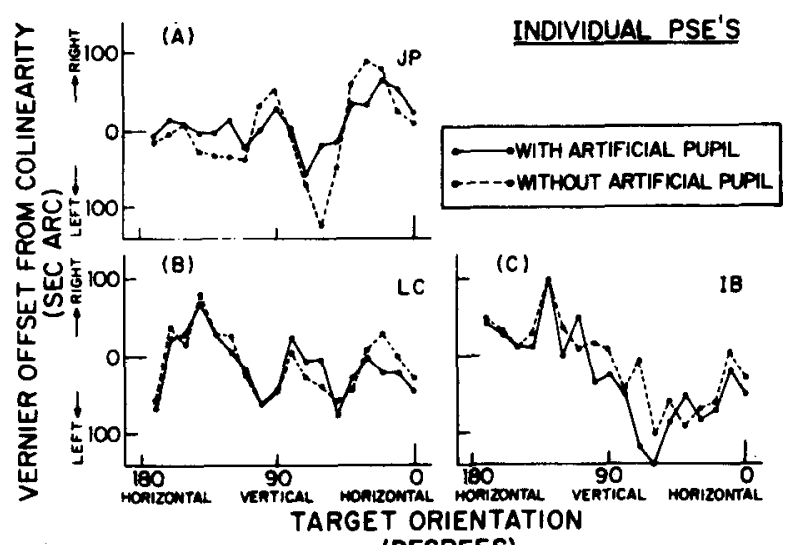
(DEGREES)

Figure 5. PSEs for three subjects (J.P., L.C., and 1.B.) shown for the unlimited viewing condition as a function of target orientation. In each panel, data are shown with (solid lines) and without (dashed lines) the artificial pupil. 


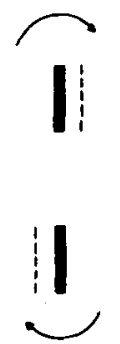

(a)
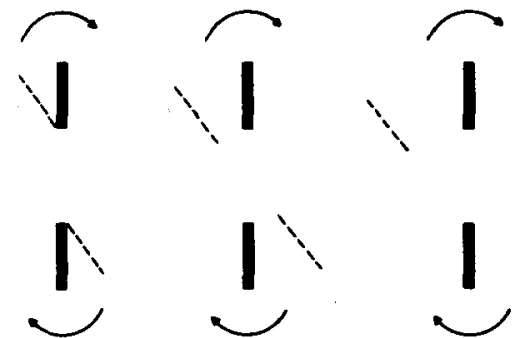

(b)

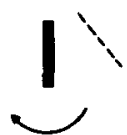

(c)

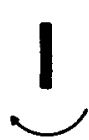

(d) physical position -... perceived position

Figure 6. Representation of spatial induction hypothesis for a clockwise rotating target. Prediction of displacement as a result of figural aftereffect in (a), predictions from tilt induction with "centers" at the ends of the lines in (b), at the center of rotation in (c), and below the rotation center in (d).

appearance of offset with which we are concerned. Such induction effects are particularly prominent both immediately before and immediately after the onset of inducing stimuli relative to their magnitudes when an inducing stimulus has been present for a long period. In order for the induction effect to predict the rotation-contingent offset, it is necessary that the transient enhancement of displacement be asymmetrical around the time of onset of the inducing line. If the induction was produced by figural aftereffect displacement, the enhancement would have to be larger for an inducing line whose onset occurs prior to that of the test line than for an inducing line whose onset follows the test flash (preinduction dominant) (Figure 6a). On the other hand, if the induction were due to the mechanism involved in tilt induction, the reverse temporal asymmetry would correctly predict the obtained vernier offset; in this case the enhancement would have to be larger for an inducing line whose onset occurs after the test flash (postinduction dominant) (Figure 6b).

It should be noted that the representation of the influence of tilt induction in Figure $6 \mathrm{~b}$ assumes that for induced tilt the perceived position rotates around the physical point corresponding to the end of the line itself. If the induction were centered around the center of rotation of the line pair, no vernier offset would result (Figure 6c). If the induction were centered around a point below the physical center of rotation of the line pair, the same temporal asymmetry of the transient enhancement as was assumed in Figure $6 \mathrm{~b}$ (postinduction dominant) would predict the wrong direction of vernier offset (Figure 6d), but the reverse asymmetry (preinduction dominant) would predict the correct direction.

\section{Latency}

Since points at the far ends of the rotating bars sweep out circles of larger radius than points nearer the center of the target, the energy/time stimulating a point nearer the end will be less than the ene"gy/time stimulating a point nearer the center. Since response latency is shorter for stimuli of increasec energy (cf. Bowen, Pola, \& Matin, 1974; Lit, 1968), points nearer the outer ends of the lines would be expected to be perceived later than points nearer the cente:. This suggests that when the lines are in the positions shown by the dark bars in Figure $6 \mathrm{~b}$, their perceived orientations will be displaced in the direction of the dashed lines, thus yielding the vernier displacement. $^{2}$

The induction hypothesis and the latency hypothesis both predict the data in the present report. Both mechanisms would be expected to depend on stimulus velocity, although in somewhat different ways. However, while the induction hypothesis readily predicts offsets of greater magnitude during rotation for subjects whose range of stationary offsets is larger, as in Figure 4 , it is not yet clear that the latency hypothesis can make a similar prediction.

\section{REFERENCE NOTE}

1. Boff, K., Matin, L., \& Pola, J. An illusion of vernier offset produced by rotary target motion. Presented at the annual meeting of the Eastern Psychological Association. New York. April, 1975.

\section{REFERENCES}

Bouma, H., \& ANDriessen, J. J. Induced changes in the perceived orientation of line segments. Vision Research, $1970,10,333-350$.

Bowen, R. W., Pola, J., \& Matin, L. Visual persistence: Effects of flash luminance, duration and energy. Vision Research, 1974, 14, 295-303.

Finney, D. S. Probit analysis. Cambridge, England: Cambridge University Press, 1947.

GrarAM, C. H. Visual form perception. In C. H. Graham (Ed.), Vision and visual perception. New York: Wiley, 1965.

Kohler. W., \& Wallack. H. Figural after-effects: An investigation of visual processes. Proceedings of the American Philosophical Society. 1944, 88, 269-357.

LEIBOwITZ. $H$. Some factors influencing the variability of vernier adjustments. American Journal of Psychology. 1955. 68. $266-273$.

LrT. A. Illumination. effects on depth discrimination. The Optometric Weekly, 1968, 59, 42-55.

Matin. E. Light adaptation and the dynamics of induced tilt. Vision Research, 1974, 14, 255-265.

Matin, E.. Matin, L., Hirsch, J., \& Kornheiser, A. The dynamics of induced tilt. Transactions of the American Acudemy of Optometry. 1972, 49, 897.

\section{NOTES}

1. A theoretical analysis of the correlations in Figure 4 and others derived from the present data is under way and will be presented elsewhere (Main \& Boff, in preparation).

2. Protessor Joel Pokorny of the Eye Research Laboratories. University of Chicago, suggested this interpretation involving latency.

(Received for publication October 27, 1975; revision accepted January $2,1976$. ) 